\title{
ROAD NAIL: EXPERIMENTAL SOLAR POWERED INTELLIGENT ROAD MARKING SYSTEM
}

\author{
Dragan Samardžija ${ }^{*}$ - Nikola Teslić ${ }^{*}$ - Branislav M. Todorović ${ }^{* *}$ \\ Erne Kovač $^{* *}$ — Dorde Isailović ${ }^{* *}$ - Bojan Miladinović ${ }^{* *}$
}

\begin{abstract}
Driving in low visibility conditions (night time, fog or heavy precipitation) is particularly challenging task with an increased probability of traffic accidents and possible injuries. Road Nail is a solar powered intelligent road marking system of wirelessly networked signaling devices that improve driver safety in low visibility conditions along hazardous roadways. Nails or signaling devices are autonomous nodes with capability to accumulate energy, exchange wireless messages, detect approaching vehicles and emit signalization light. We have built an experimental test-bed that consists of 20 nodes and a cellular gateway. Implementation details of the above system, including extensive measurements and performance evaluations in realistic field deployments are presented. A novel distributed network topology discovery scheme is proposed which integrates both sensor and wireless communication aspects, where nodes act autonomously. Finally, integration of the Road Nail system with the cellular network and the Internet is described.
\end{abstract}

K e y w o r ds: road safety, smart roads, wireless sensor network, vehicle-to-infrastructure

\section{INTRODUCTION}

In last few years, extensive research and a number of innovations in the field of improved road safety have been reported: intelligent infrastructure [1-3], vehicular networking [4-7] and in- vehicle solutions [8]. Rapid improvements and wide application of semiconductor products have been the key enablers of effective implementation of sensor, wireless and battery technologies [9]. However, visibility is of key importance to road safety.

Roads are traditionally marked with road studs based on passive reflectors, called cat's eyes. This system operates by reflecting a portion of the light from the vehicle headlights back to the driver. In low visibility conditions, passive reflectors receive reduced light intensity from vehicle headlights and hence the intensity of reflected light is insufficient for reliable road-edge marking. In such conditions, conventional cat's eyes road studs are not safe and reliable method of delineation, since they rely on headlight reflection. More than a decade ago, powered road studs have appeared as option for improved road safety [10-12]. Solar powered intelligent road studs are hot topic of interest in academia [10,12], government [13] and industry [14]. Such a system demonstrates a 10-fold greater visibility of road delineation compared to conventional system $[13,14]$. Reaction time of a motorist driving at a speed of $100 \mathrm{~km} / \mathrm{h}$ is predicted to be over 30 seconds. That's a dramatic difference compared with conventional reflective road studs which offer only 3.2 seconds [14]. That gap can be overcome by using intelligent road studs, which provide an additional "preview" time for drivers on winding roads. At their UK installation sites, intelligent road studs have already reduced night time accidents by over $70 \%[14]$.

In this paper we present an advanced experimental solar powered system for intelligent marking of the road edge during low visibility. The system is named Road Nail, as originally partly presented in [15].

This study combines the most advanced solutions in sensor, wireless communication and battery-powered technologies to design and implement a novel road-safety system. It consists of an array of nodes that are installed on both sides of the road, being mutually separated by a few tens to a few hundreds of meters. Each node consists of (i) vehicle detection subsystem, (ii) light signalization subsystem, (iii) wireless communication and control subsystem, and (iv) power supply subsystem, with solar panels and a battery. During the day, nodes have their batteries recharged, while during low visibility and/or night time they switch to active mode enabling detection of passing vehicles and timely activation of the light signalization. Upon detection of a passing vehicle, the node generates a wireless message. After the detection or reception of a message indicating that a nearby node detected a vehicle, the signalization lights should be turned on (either red or white depending on the side of the road). In order to save battery power the lights will be turned off after a predefined period of time.

The nodes are installed on both sides of the road, not requiring disruption of traffic during the installation and maintenance. Additionally, the nodes do not require infrastructure power. Furthermore, this system may be connected to the global Internet via a specific gateway. This would allow local node information to be forwarded

\footnotetext{
* Faculty of Technical Sciences, University of Novi Sad, Trg Dositeja Obradovića 6, 21000 Novi Sad, Serbia, \{Dragan.Samardzija; Nikola.Teslic\}@rt-rk.uns.ac.rs; ${ }^{* *}$ RT-RK, Institute for Computer Based Systems, Narodnog Fronta 23A, 21000 Novi Sad, Serbia, \{Branislav.Todorovic; Erne.Kovac; Djordje.Isailovic; Bojan.Miladinovic\}@rt-rk.com
} 


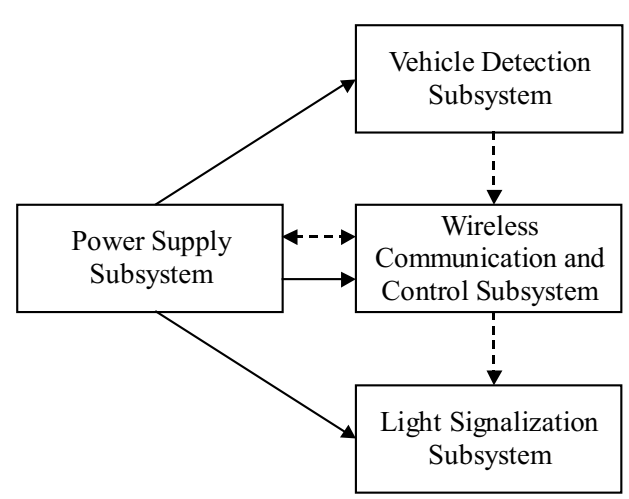

Fig. 1. Block diagram of the Road Nail signaling device

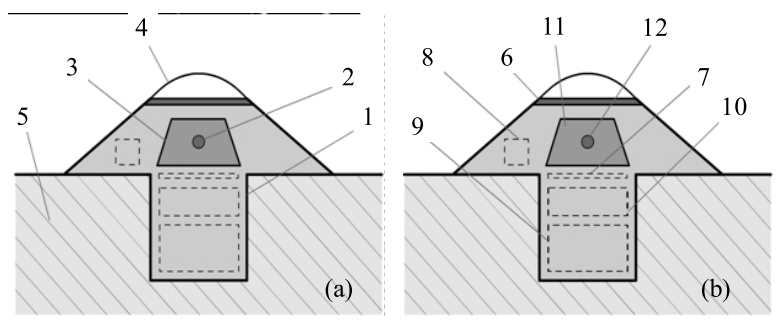

Fig. 2. Road Nail node: (a) - Front view, (b) - Back view

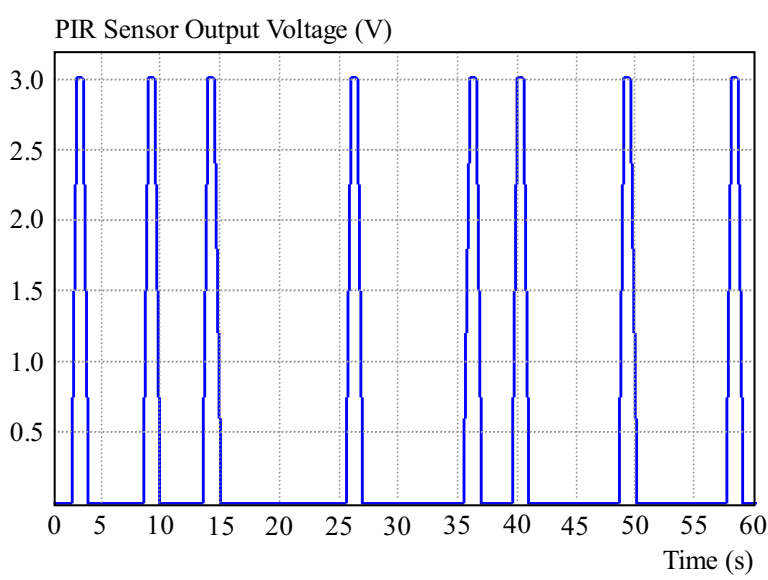

Fig. 3. PIR detector output as a function of time with vehicles passing at $t=3,9,14,26,36,40,49$ and 58 seconds

to a remote control and monitoring center where road and node conditions may be monitored. In addition, the control and monitoring center can send different information to all or individual nodes in the area. This represents a flexible platform for a versatile implementation of the infrastructure-to-vehicle (I2V) and vehicle-toinfrastructure (V2I) concept [16-18].

In Section 2 we describe the node architecture and implementation. Performance and power consumption of the sensor, wireless communication and control, light signalization and power subsystem are presented. In Section 3 we describe the network organization and system functionality where a novel distributed network topology discovery scheme is proposed. It integrates both sensor and wireless communication aspects, where nodes act au- tonomously. In addition, a detection scheme that relies on radio transmission is proposed. We describe integration with the cellular network and the Internet in Section 4 and conclude in Section 5.

\section{SIGNALING DEVICE ARCHITECTURE AND IMPLEMENTATION}

Each signaling device, $i e$, node, in the Road Nail system consists of four subsystems, as shown in Fig. 1. The dashed lines depict flow of information and control, while the solid lines correspond to power supply.

The Road Nail node implementation is shown in Fig. 2. The upper part of the signaling device (4) is made of a transparent, waterproof material (eg, glass) allowing passing of the solar light to the solar cell (6) and protecting electronic components $(7,8,9$ and 10) from rain or snow. On the front and back side of the housing, there are signalization light sources (2 and 12), with reflective backgrounds (3 and 11). The red and white signalization lights are on the front and back side of the node, respectively. As given in Fig. 2, the nodes may be installed such that the lower part of the device (1) is in the ground (5). Alternatively, the nodes may also be installed above the ground (eg, typically $80 \mathrm{~cm}$ above the ground), where additional mechanical support structure is needed. Inside the housing there is a printed circuit board with a microcontroller and a wireless communication transceiver (7), a number of sensors (8) and a battery (9) with its management (10).

\subsection{Vehicle Detection Subsystem}

The sensor, $i e$, vehicle detection subsystem consists of a set of sensors which provide information to the wireless communication and control subsystem. In this particular implementation we applied (i) a motion sensor based on passive infrared (PIR) technology and (ii) a light sensor. The PIR sensor [19] is used to detect moving vehicles. This particular sensor has been proven very efficient with the detection range of approximately 10 meters, and low power consumption of less than $15 \mathrm{~mW}$. Using extensive measurements the sensor sensitivity is set such that the probability of missed detection is approximately $P_{M}=0.1$. The probability of false alarm is approximately $P_{F}=0.005$. The measurements were performed in realistic night time conditions, on a suburban road with moderate traffic density, with the vehicular speed limited to $60 \mathrm{~km} / \mathrm{h}$. For example, in Fig. 3 we present the output voltage as a function of time when vehicles passed at $t=3,9,14,26,36,40,49$ and 58 seconds.

In addition, we applied a light sensor [20] for detection of vehicle headlights with a power consumption of approximately $1 \mathrm{~mW}$. The detection threshold is set such that the probability of missed detection is approximately $P_{M}=0.1$, with the negligible false alarm probability (less 


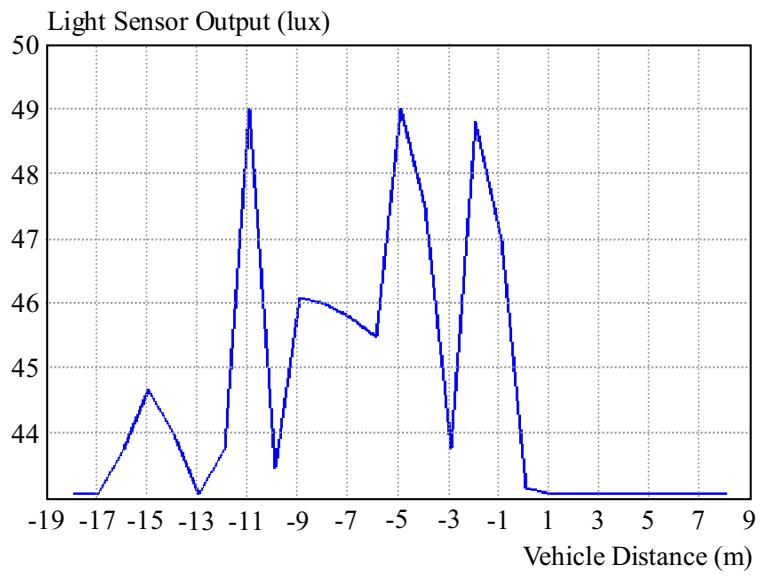

Fig. 4. Light sensor output as a function of distance between the passing vehicle and the node

than 0.005). As an example, in Fig. 4 we present the sensor output as a function of the approaching vehicle distance, with the headlights on. The measurements were performed during night time on a road with no other significant light source. The exact light sensor response depends on the speed, height and headlight intensity on the passing vehicle, where Fig. 4 corresponds to one such case.

The light sensor is directive. Namely, it is directed towards direction vehicles are coming from, with respect to the side of the road the particular node is installed on. The directivity may be also noted in Fig. 4, where sensor readings drop once the vehicle has passed by the node (ie, at distance of 0 meters and beyond). Therefore, in combination with the PIR sensor, the light sensor may provide information about which direction the detected vehicle arrives from.

Note that in the case of each sensor, the probability of missed detection could be lowered by adjusting the detection threshold level. However, it would result in a higher false alarm probability which is not desirable [21]. Namely, it would cause a more frequent activation of other subsystems resulting in higher overall power consumption. Using the sensor outputs the wireless communication and control subsystem performs the following system functions:

A. Activation of the light signalization - Upon detection of a passing vehicle, the light signalization should be turned on, marking the edge of the road. A further refinement may be implemented using the information on the direction of the moving vehicle. For example, if the directive light sensor detects an approaching vehicle, the node infers that the vehicle and node are on the same side of the road. Consequently, instead of turning both the red and white signalization light on, only the red is turned on, providing additional power savings. Simultaneously, in the pair node on the opposite side of the road, the white signalization light is turned on.

B. Generation of wireless messages - Upon detection of a passing vehicle, the node generates the corresponding wireless message, thus informing neighboring nodes about the detection. Each node that successfully have received that message autonomously decides whether to turn its signalization light on. The decision will be based on the existing knowledge of the network topology (to be addressed in Section 3).

C. Decision on operational mode - In addition to detecting vehicle headlights, the light sensor may be used to detect the amount of the background illumination. If the long term average of the illumination level is high, each node may enter a hibernation mode. While in the hibernation mode, no light signalization or wireless messaging is activated, thus significantly lowering power consumption. This would correspond to daytime and good visibility. However, if the average illumination level is low, the node is in an active mode. While in the active mode, the light signalization may be turned on, and the corresponding wireless messages generated. This would correspond to night time and/or low visibility.

D. Network topology discovery - Details are provided in Section 3.

During experimentation, we noticed that the performance of PIR and light sensors diminished as the dirt accumulated covering the subsystem housing. The effects were particularly severe when the node was installed such that its lower part was in the ground. As expected, with the node installed above the ground $(\mathrm{eg}, 80 \mathrm{~cm}$ above the ground), the accumulation of dirt was significantly lower, and the performance degradation was marginal.

To address the above problem, in Section 3 we propose an alternative vehicle detection scheme that is based on radio transmission (ie, implementing a passive radar concept using the existing wireless communication transmission).

\subsection{Light Signalization Subsystem}

The light signalization subsystem consists of a set of light-emitting diodes (LEDs) and an LED driver. The LEDs are used because of their low power consumption while having satisfactory brightness providing a clear and visible marking of the road edge in low visibility. Based on extensive analysis and measurements we conclude that this subsystem consumes a significant fraction of the available power. Specifically, on a road with high traffic density (ie, the light signalization is always on), it accounts for more than $60 \%$ of overall power consumption. The measurement results are presented in Fig. 5.

\subsection{Wireless Communication and Control Sub- system}

The wireless communication and control subsystem is based on a wireless transceiver and microcontroller, all integrated in a chip [22]. It is in charge of exchanging messages between the neighboring nodes over the wireless interface, as well as sensor output analysis and battery state control. The wireless network interface is based on 


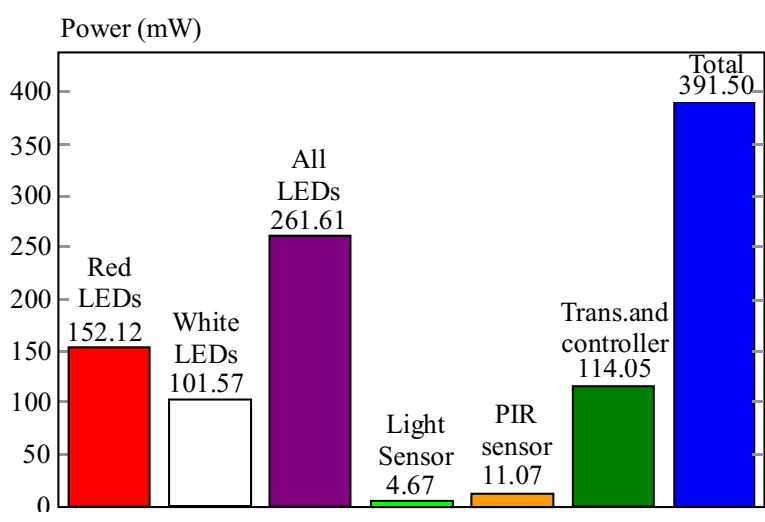

Fig. 5. Power consumption measurement results
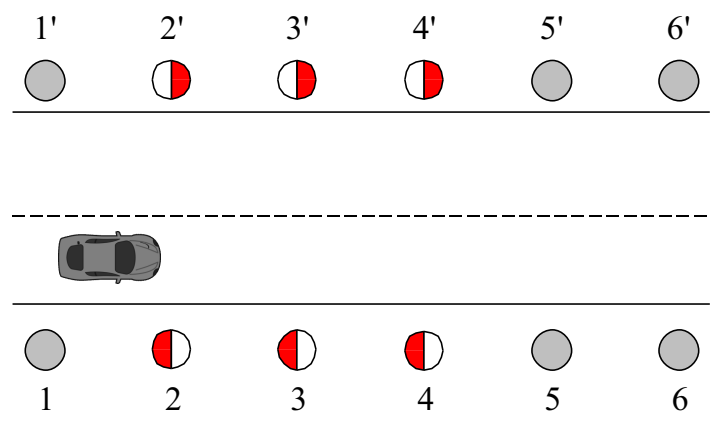

Fig. 6. Road Nail system

the IEEE 802.15.4 standard [23] which defines the physical and medium access control layer for personal area network (PAN). The standard applies carrier sense multiple access with collision avoidance (CSMA/CA) mechanism and supports star as well as peer-to-peer topologies. The implemented protocol ensures features such as low complexity, cost, power consumption, and low transmission data rates. Low power consumption is the most important feature in this application due to limited amount of accumulated energy. During daytime and good visibility, it enters the ultra low-power hibernation mode. While in the active mode (night time and/or low visibility), on a road with high traffic density (ie, a wireless message is transmitted every $10 \mathrm{msec}$ ), it consumes approximately $25 \%$ of overall power. The measurement results are presented in Fig. 5.

\subsection{Power Supply Subsystem}

The power supply subsystem consists of the following three segments: solar cells, rechargeable battery and battery management. The solar cells provide enough energy for full functionality of the signaling device over some predefined period of time. The battery management is in charge of monitoring and control of all battery parameters and processes including battery charging and discharging. We have conducted a number of laboratory and field measurements. The power consumption measurement results are presented in Fig. 5. The measurements are performed during night time, at around $15^{\circ} \mathrm{C}$. As said earlier, it was on a road with high traffic density. For the given power consumption, we have analytically and experimentally confirmed that the fully charged battery could support almost 20 hours of the continuous operation (in the active mode, without battery recharging during that period).

In order to meet projected power consumption, three solar modules are used each being $66 \times 95 \times 6$ millimeters in size [24]. Extensive measurements have been performed during cloudy and sunny atmospheric conditions, with different sun incidence angles. Our analysis indicates that in order to have a continuously operational system, in a period lasting four days, it is sufficient to have an aggregate of 10 sunny hours enabling proper recharging of the battery (on average, 2.5 sunny hours a day).

In addition to solar power other energy sources are to be investigated. For example, air-flow and vibration energy sources are the subject of our future work.

\section{NETWORK ORGANIZATION AND SYSTEM FUNCTIONALITY}

A unique address is assigned to each node. The addresses are allocated at the medium-access control layer (eg, Ethernet MAC address in IEEE 802.15.4 [23]) or network layer (eg, IP address in 6LoWPAN [25]). Each message contains the transmitting node address (ie, source address). In addition, a message may be sent to a particular node, thus containing the corresponding destination address, or broadcasted to all the nodes within the communication range. Each node can decode the information content of received messages, $i e$, no ciphering or common ciphering is applied in the system.

Figure 6 illustrates the basic installation and functionality of the system. The distances between the nodes depend on the conditions of the road. For example, sharper curves would require denser installation of the nodes. As given in Fig. 6, a vehicle is detected for the first time by node 1. After detection, it turns its red signalization light on and transmits an appropriate message. The message is received by the neighboring nodes. Upon reception of the message, nodes 2 and 2' turn the red and white signalization lights on. The message is then forwarded, enabling other nodes to turn the signalization on (eg, nodes 3,3 , 4 and 4') or wait for future detection events (eg, nodes 5 and 5' may wait for node 2 or 2' to detect the vehicle). As soon as the vehicle passes by node 1 , in order to conserve energy, it turns its signalization light off. The process continues in the direction of the vehicular movement, resulting in a wave-like propagation of the signalization light along the road.

Each node is expected to send a very limited amount of information, transmitting only a few messages per second. Considering that there is a limited number of nodes in the given area, probability of collisions between the transmissions will be very low. Occasional collisions will be 
resolved by the CSMA/CA mechanism. In other words, this communication system will operate significantly below its communication capacity limits. However, due to the low transmit power and poor receiver sensitivity; this system will be primarily coverage-limited, which is analyzed in Section 4.

\subsection{Network Topology Discovery}

A node turns its signalization on only if its immediate neighbors have generated a message indicating detection of a passing vehicle, while ignoring messages received from the nodes which may be at a greater distance, or on adjacent roads (eg, as on intersections). In order to properly act upon message reception, each node must have knowledge of relative positions, ie, topology of other nodes in the area. A trivial solution is to use a priori known arrangement of nodes, ie, a fixed topology where the physical location and address of each node in the system are known to other nodes. However, since additional nodes may be installed during future system expansions, or as replacements for older malfunctioning nodes, an adaptive topology discovery solution is highly desirable. One such solution is described in the following.

In order to describe the topology, each node creates a list of nodes that it is able to directly communicate with, including information about the quality of each wireless link, ie, received signal power. At time instant $l$, for node $i$, an estimate of the received signal power corresponding to node $j$ is

$$
\bar{P}_{i, j}^{r x}(l)=\frac{1}{N_{m}} \sum_{n=1}^{N_{m}} \widetilde{P}_{i, j}^{r x}(l-n+1)
$$

where $\bar{P}_{i, j}^{r x}(l)$ is the power estimate for the message received at time instant $l$ and the averaging is performed over the $N_{m}$ most recent messages. Each node in the system periodically broadcasts messages (eg, twice per second), enabling other nodes to measure the corresponding received power and update the above statistics.

For example, using the above statistics the immediate neighbors may be selected based on the received signal power, relative to the signal powers of other nodes within the communication range. However, this may not be appropriate. For example, nodes installed on adjacent roads or intersections may be at short distances from each other, having high wireless link quality. Nevertheless, messages generated by the nodes that indicate vehicle detection on adjacent roads should be ignored, ie, the signalization should not be turned on.

As said earlier, each device also generates and sends messages upon detection of a passing vehicle. As an alternative solution, those messages and time intervals between them are used to determine relative position of each node in the area. Namely, if two nodes consistently detect a passing vehicle and exchange the corresponding wireless messages within a predefined time period, they will be considered immediate neighbors belonging to the same road. On the contrary, if the signal quality is high, but detection of a passing vehicle by one node is not consistently followed or preceded by the other, it will be assumed that the nodes belong to different roads, eg, on an intersection. To estimate relative distances between node $i$ and other nodes the following algorithm is implemented. In general, the node indices are uniquely associated with their addresses (eg, either Ethernet MAC or IP address).

1 . Node $i$ records time instant $\tau_{i, j}$ when a message arrives from node $j(j \neq i)$ indicating that it detected a passing vehicle. In order to estimate time instant $\bar{\tau}_{i, j}$, when a vehicle is detected by node $j$, the expected message transmission interval $T_{D}$ is subtracted as,

$$
\bar{\tau}_{i, j}=\tau_{i, j}-T_{D}
$$

$T_{D}$ is the time interval, $i e$, latency required to perform transmitter processing, over-the-air transmission and receiver processing. Note that a more elaborate estimation in (2) may be used to better address possible multi-hop message forwarding.

Only the records of the most recent detection events are stored, not exceeding a predefined time interval $T_{w}$. Namely, at time instant $t, t-\bar{\tau}_{i, j} \leq T_{w}$. For example, in our implementation $T_{w}=6 \mathrm{sec}$, where for a moderate vehicle speed of $60 \mathrm{~km} / \mathrm{h}$ it corresponds to the path interval of 100 meters. Node $i$ executes this step until it detects a passing vehicle itself.

2 . At time instant $t_{0}$, node $i$ detects a passing vehicle, and waits additional $T_{w}+T_{D}$, while recording all possible detections within the time interval $\left[t_{0}-T_{w}, t_{0}+T_{w}\right]$. After this interval node $i$ executes the following step.

3. Based on the recorded detection moments during the interval $\left[t_{0}-T_{w}, t_{0}+T_{w}\right]$, each node $j(j \neq i)$ is mapped to an index corresponding to its relative distance from node $i$. Namely, if the absolute difference $\left|t_{0}-\bar{\tau}_{i, j}\right|$ is the $p$-th smallest compared to the other recorded detection moments, the relative distance between nodes $i$ and $j$ is

$$
d_{i, j}(k)=p
$$

where $k$ is the index of interval $\left[t_{0}-T_{w}, t_{0}+T_{w}\right]$. If multiple detection events are reported by node $j$, the one corresponding to the smallest $p$ is adopted. However, if node $j$ has not reported any detection event, its relative distance is set to a default value $D_{\max }$, assuming that it may be farthest from node $i$.

Using the relative distance in expression (3), for time interval $k$, the average value is obtained as

$$
\bar{d}_{i, j}(k)=\frac{1}{L} \sum_{i=1}^{L} d_{i, j}(k-l+1)
$$

with the averaging performed over the $L$ most recent intervals $\left[t_{0}-T_{w}, t_{0}+T_{w}\right]$, each corresponding to node $i$ detecting a passing vehicle. With respect to node $i$, the 


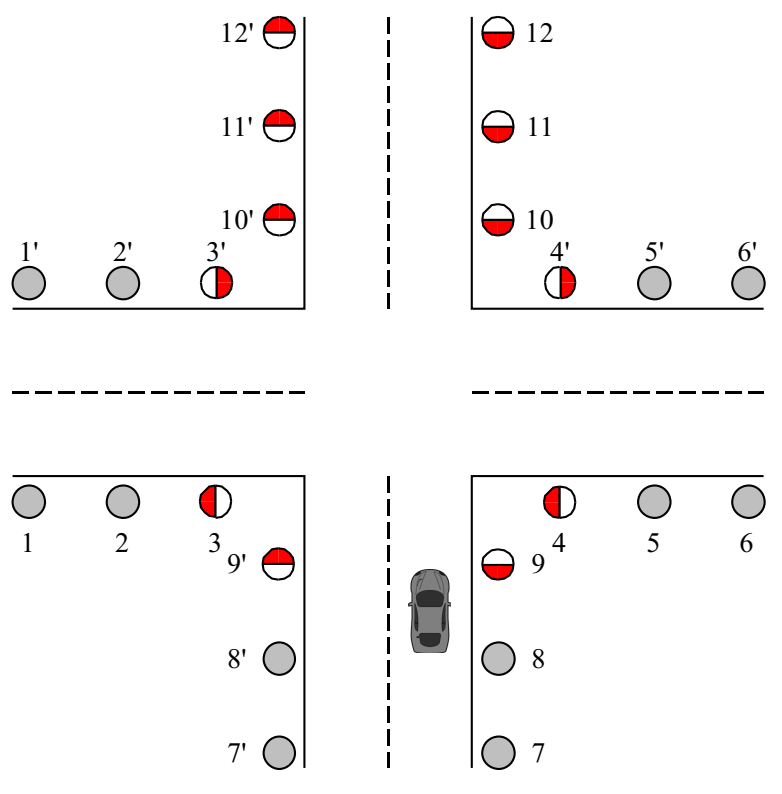

Fig. 7. Intersection with the Road Nail system

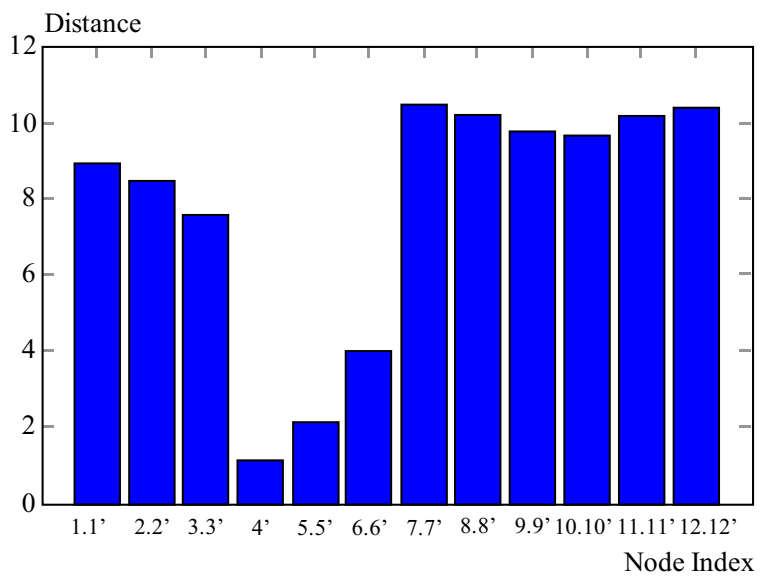

Fig. 8. Average relative distance in expression (4), with respect to node $4, P_{M}=0.1, D_{\max }=12$

closest node $o_{i}$ is determined as the one with the minimum average relative distance

$$
o_{i}(k)=\min _{j=1, \ldots, N, j \neq i} \bar{d}_{i . j}(k)
$$

where $N$ denotes the number of neighboring nodes. Typically, the closest node would be a pair node installed on the opposite side of the road. For example, in Fig. 6, with respect to node 4 , its pair node is node 4'. Further, the second and third closest nodes correspond to the second and third smallest average distances in expression (4), respectively. For example, in Fig. 6 each node determines up to nine closes nodes that belong to the same road, ie, the immediate neighbors. The nine nodes are expected to correspond to one pair node on the opposite side of the road, and four nodes up and four nodes down the road. For example, with respect to node 4 , the immediate neighborhood consists of nodes 2, 2', 3, 3', 4', 5, 5', 6 and 6 '. Upon a vehicle being detected by any node in its immediate neighborhood, node 4 will turn its light signalization on.

4. Once node $i$ determines a number of closest nodes that belong to the same road, it may also decide on its relative position on the road. Namely, whether the neighboring nodes are up or down the road from each other with respect to node $i$. For example, let nodes $m$ and $n$ belong to the node $i$ immediate neighborhood. The following statistics is used to determine their position.

$$
s_{i, n, m}(k)=\operatorname{sgn}\left[\left(t_{0}-\bar{\tau}_{i, n}\right)\left(t_{0}-\bar{\tau}_{i, m}\right)\right]
$$

where $k$ is the index of the time interval $\left[t_{0}-T_{w}, t_{0}+T_{w}\right]$. For time interval $k$, the average value is obtained as

$$
\bar{s}_{i, n, m}(k)=\operatorname{sgn}\left(\frac{1}{L} \sum_{i=1}^{L} s_{i, n, m}(k-l+1)\right)
$$

with the averaging performed over the $L$ most recent intervals. Using (7) the decision is made as

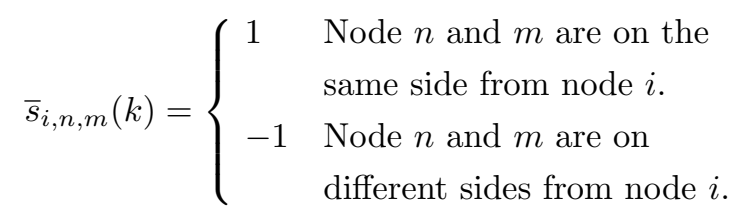

For example, in Fig. 6, with respect to node 4, $\bar{s}_{4,2,3}(k)=1$ while $\bar{s}_{4,2,6}(k)=-1$.

Each node executes the above algorithm autonomously. The changes in the topology are continuously tracked, and the topology information is obtained from expressions (4) and (7). Information about the immediate neighbors and/or their relative positions may be used to implement different rules on how a node should act upon reception of messages from other nodes ( $\mathrm{eg}$, to turn the signalization light on or off).

We consider the above topology discovery scheme novel because it integrates both sensor and wireless communication aspects. Furthermore, it is a fully distributed scheme, where each node acts autonomously. Unlike other well-know schemes $(e g,[26,27])$, the proposed scheme does not require any specific communication protocol that would be exclusively used to enable topology discovery. We consider those characteristics particularly suitable for the proposed intelligent road marking system where nodes may be added, replaced or removed without explicit reconfiguration of the network.

To illustrate the performance and behavior of the above algorithm we consider an intersection with the nodes installed as in Fig. 7. The numerical analysis is performed under the following assumptions. The average vehicular speed is $30 \mathrm{~km} / \mathrm{h}$, with a vehicle arriving every 30 seconds. With equal probability, a vehicle may go though the intersection or make a left or right turn. On the same road, inter-node distance is 50 meters, with the closest nodes installed 10 meters from the intersection 


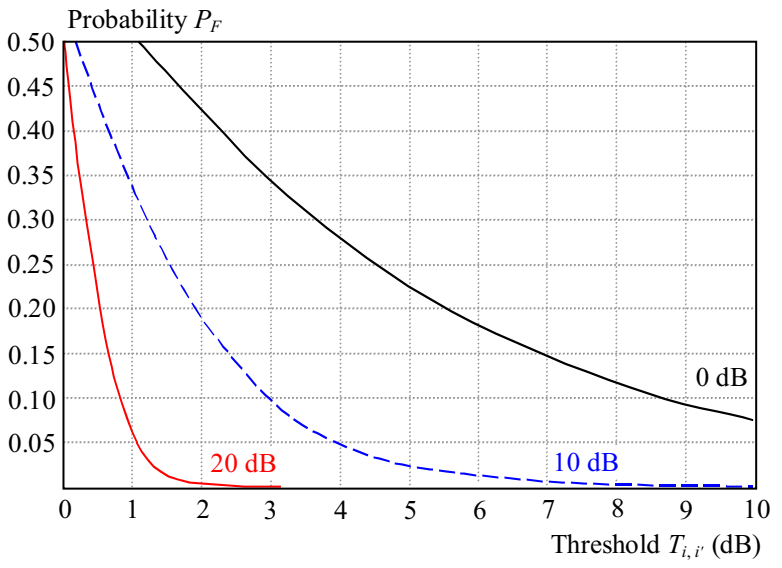

Fig. 9. False alarm probability versus the threshold $T_{i, i^{\prime}}$, for different levels of SNR, $N_{m}=100$

(Figure 7 is not up to scale). The probability that a node may miss detection of a passing vehicle is $P_{M}=0.1$, with a negligible false alarm probability ( $e g$, similar to the PIR sensor in Section 2). For the sake of simplicity, the nodes in Fig. 7 can directly communicate with each other.

The average relative distances in expression (4) with respect to node 4 are presented in Fig. 8, for $D_{\max }=12$. For example, note that node 9 is physically closer to node 4 than node 2 . However, in terms of the topology and relative distance, node 2 is closer to node 4 . This is a consequence of higher probability that the same vehicle passes by both node 2 and 4 , rather than node 9 and 4 , rendering node 2 closer in terms of the network topology.

The algorithm selects the immediate neighbors based on the smallest average relative distance. Which nodes will be selected may depend on the deployment strategy. If for example, nodes 4', 5, 5', 6, 6', 3 and 3' are selected, the node 4 light signalization will be turned on only if a vehicle is detected on the road it belongs to. This may result in a less frequent activation of the signalization and lower power consumption. However, this strategy makes no attempt to assist vehicles that may be making a turn on the intersection. On the other hand, if nodes 4', 5, 5', $6,6^{\prime}, 3,3$ ', 2, 2', 1, 1', 9, 9', 10 and 10' are selected, node 4 will turn its signalization on, even if a vehicle is not on its road, as it enters the intersection. This would assist vehicles making a turn. This however results in a more frequent activation of the signalization and higher power consumption. One such situation that corresponds to the latter selection and deployment strategy is presented in Fig. 7. In this case the vehicle that is entering the intersection causes the signalization on the adjacent roads (node 3, 3', 4 and 4') to be turned on, in anticipation that the vehicle may make either a left or right turn.

Furthermore, since the presented algorithm is adaptive, after an initial training period, a newly added or replaced node would obtain accurate estimates of the average relative distances in expression (4). Once the accurate estimates are available, the node becomes operationally integrated with the rest of the system ( $\mathrm{eg}$, for the system in Fig. 7, typically after 100 vehicles are being detected).
Upon installation of a new node, no explicit reconfiguration of the existing nodes is needed. They would learn about the change in the topology using the same adaptive process.

The above algorithm was implemented and tested under realistic deployment scenarios. A single road and intersection deployment have been evaluated. The behavior and performance closely followed the above analysis.

\subsection{Vehicle Detection Using Wireless Transmis- sion}

As previously described, radio transmission is used to implement exchange of messages between the nodes, $i e$, it implements the wireless communication protocol between the nodes. In this subsection, we propose an auxiliary application of that radio transmission. Namely, it will be used to detect a passing vehicle.

As described in Section 2, the vehicle detection subsystem integrates a number of conventional sensors (eg, PIR and light). Power consumption, size, cost and performance (eg, the PIR and light sensor sensitivity is impacted by dirt) are critical aspects of that subsystem. The solution as proposed in this subsection would (i) improve the detection performance in combination with the existing conventional sensors (eg, lower probability of missed detection and false alarm) and/or (ii) remove a need for application of conventional sensors resulting in lower power consumption, size and cost.

The solution is based on a premise that a presence of a vehicle in the vicinity of a node would significantly alter its radio propagation environment. Therefore, in order to facilitate this solution, each node periodically broadcasts a message (eg, twice a second). Upon reception of the message from node $j$, at time instant $l$, node $i$ estimates the received power $P_{i, j}^{r x}(l)$. The power is compared against the average power which is estimated as given in expression (1). This is essentially a radar which is using the existing radio transmission originally intended for communication purposes. It may be classified as passive radar [28], with a novel application- specific short-range implementation.

As a simplest implementation, node $i$ monitors the received power corresponding to its pair node $i^{\prime}, e g$, node 2 and 2 ' in Fig. 6. If the difference between the average and received signal power exceeds a specific threshold, node i decides that a vehicle has passed between the two nodes. In other words,

$$
\begin{aligned}
& \frac{\bar{P}_{i, i^{\prime}}^{r x}(l-1)}{\widetilde{P}_{i, i^{\prime}}^{r x}(l)}= \\
& \quad\left\{\begin{array}{l}
\geq T_{i, i}, \quad \text { vehicle is detected. } \\
<T_{i, i}, \quad \text { No vehicle, perform averaging in (1). }
\end{array}\right.
\end{aligned}
$$

where $T_{i, i^{\prime}}$ is a threshold. The threshold is set such that the desired probabilities of false alarm and/or missed 


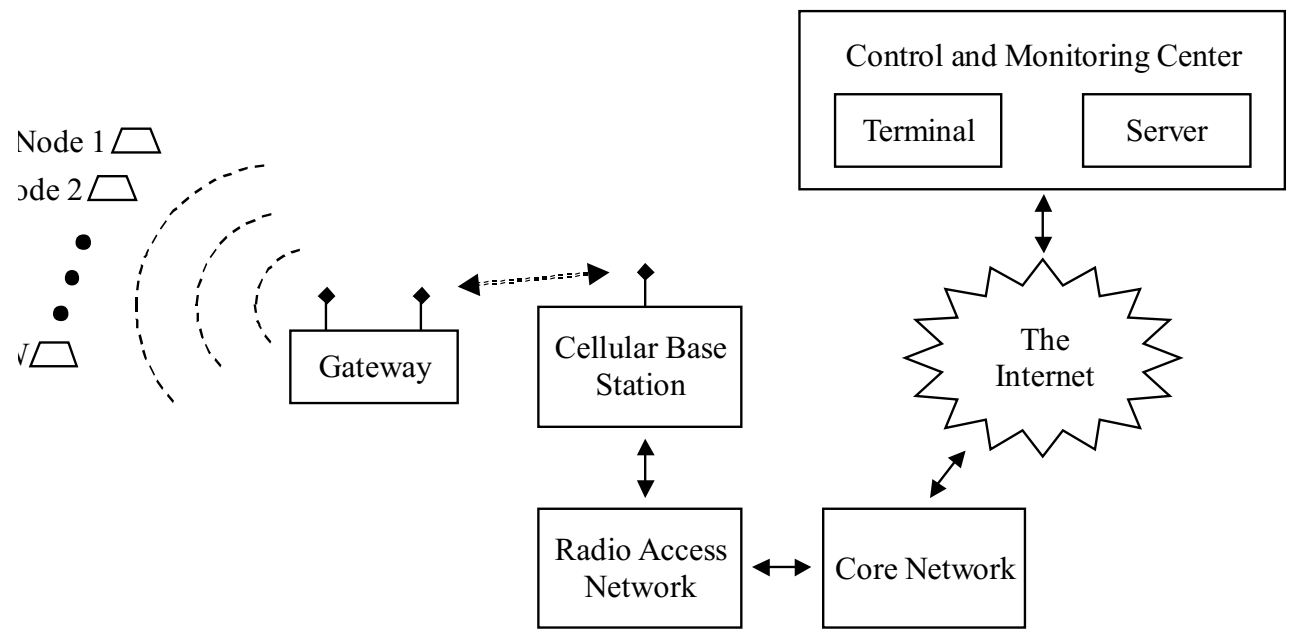

Fig. 10. Road Nail system integrated with the Internet and the control and monitoring center

detection are achieved. The above criterion assumes that a vehicle would cause attenuation.

Using numerical analysis, in Fig. 9 we present the probability of false alarm as a function of the threshold $T_{i, i^{\prime}}$, for different levels of signal-to-noise ratio (SNR). The SNR is defined as

$$
S N R_{i, i^{\prime}}=10 \log _{10} \frac{P_{i, i^{\prime}}^{r x}}{N_{0}}(\mathrm{~dB})
$$

where $P_{i, i^{\prime}}^{r x}$ is the received signal power (noise-free, no vehicle is present). $N_{0}$ is the variance of the wireless channel additive white Gaussian noise (AWGN). The noise would causes false alarm events. Since each node can estimate the SNR level, it can autonomously select the threshold $T_{i, i^{\prime}}$ such that the desired $P_{F}$ is achieved. Lower threshold values result in higher sensitivity, too. Namely, for the fixed $P_{F}$, the probability of missed detection $P_{M}$ is decreasing with SNR. The wireless channel quality between two pair nodes is expected to be high, ie, having a high SNR. Therefore the vehicle detection between the two pair nodes is expected to be reliable.

The above solution have been implemented and tested on a typical suburban road. The tests were conducted during night time with light traffic (eg, on average, a vehicle arriving every 30 seconds). In the case of moderate and high SNR (above $10 \mathrm{~dB}$ ) the detection performance was exceptional. Namely, for $P_{F}=0.01$, the probability of missed detection was less than $0.5 \%$ (ie, $\left.P_{M}<0.005\right)$. In this case, the vehicle speed was limited to $30 \mathrm{~km} / \mathrm{h}$.

Initial experimentation indicated that the detection performance was not affected by the accumulated dirt. However, a detailed evaluation of this particular aspect is needed and it is a subject of our ongoing work. Considering previously discussed PIR and light sensor performance, robustness to dirt accumulation is an important feature of the above detection solution.

More elaborate schemes which track the received signal levels corresponding to multiple nodes would result in further performance improvements. For example, in Fig. 6, node 2 may monitor its pair node 2', as well as other immediate neighbors, 1, 1', 3 and 3'. Those solutions are the subject of our future work.

\section{INTEGRATION WITH THE CELLULAR NETWORK AND INTERNET}

In Fig. 10 we present integration of the Road Nail system with the cellular network and the Internet. The integration has been realized as a part of the testbed. In addition to the IEEE 802.15.4 wireless network between the nodes, a wireless link between the system and the Internet is established via a gateway and the cellular network (eg, GPRS/EDGE, UMTS or LTE). Information that is local to the nodes is available to a remote control and monitoring center. The information may be used for:

- traffic emergency monitoring (eg, a vehicle is stalled or a crash is suspected),

- speed control,

- traffic monitoring (eg, vehicles are slowing down, indicating congestion),

- road maintenance (eg, vehicles are slowing down, indicating a pothole),

- road planning (eg, detected increase in traffic density),

- emergency push button,

- node maintenance alarm (malfunctioning unit) ...

Furthermore, the control and monitoring center can send different information to all or individual nodes in the area. The nodes could transmit the received information to passing vehicles such as:

- traffic conditions,

- weather,

- work and special conditions,

- advised minimal distance between vehicles ...

As a part of the testbed, we have implemented the system in Fig. 10, providing a platform for a versatile 
implementation of the above functionalities under the I2V and V2I concept.

\section{CONCLUSIONS}

This paper presents Road Nail, an advanced experimental solar powered road marking system comprised of a wireless network of signaling devices intended to enhance driver safety. The devices are autonomous nodes that can detect approaching vehicles, exchange wireless messages with neighboring nodes and turn on a road edge safety light. The nodes are infrastructure independent, as they are powered by batteries which are charged by solar panels. The Road Nail system is especially suitable for implementation in areas where number of foggy and heavy rainy days is high and there may replace the existing signalization that is based on passive reflectors. At the first step, the system can be located at sites where, historically, visibility related accident rates are high. In addition, the presented system is suitable for implementation where road lighting is unavailable, not cost effective or environmentally not possible.

We have described our experimental testbed that consists of 20 nodes and the cellular gateway. The implementation details of the above system, including extensive measurements and performance evaluations in realistic field deployments are reported. Performance of the PIR and light sensor and power consumption of each subsystem have been provided. Just a few hours of daylight will provide enough battery power to last few days.

The proposed topology discovery scheme integrates both sensor and wireless communication aspects. It is a fully distributed scheme, where each node acts autonomously. Unlike other well- known schemes, the proposed scheme does not require any specific communication protocol that would be exclusively used to enable topology discovery. We consider those characteristics particularly suitable for the proposed intelligent road marking system where nodes may be added, replaced or removed without explicit reconfiguration of the network.

The proposed vehicle detection scheme applies the existing radio transmissions, and monitors changes in the wireless channels between the nodes. Its performance in terms of probabilities of false alarm and missed detection has been demonstrated. Initial experimentation indicated that the detection performance was not affected by the accumulated dirt.

\section{Acknowledgment}

This work is supported by the Ministry of Science and Technological Development of the Republic of Serbia under Grant TR-36029.

\section{REFERENCES}

[1] BAKKER, R.-HOGEMA, J.-HUISKAMP, W.-PAPP, Z. IRVIN - Intelligent Road and Vehicle test Infrastructure, In:
Proc. of the $8^{\text {th }}$ International IEEE Conference on Intelligent Transportation Systems, Vienna, Sep 2005, pp. 947-952.

[2] MATHUR, S.-JIN, T.-KASTURIRANGAN, N.-CHANDRASEKHARAN, J.-XUE, W.-GRUTESER, M.-TRAPPE, W.: ParkNet: Drive-by Sensing of Road-Side Parking Statistics, Proc. of the $8^{\text {th }}$ International Conference on Mobile Systems, Applications, and Services (MobiSys), San Francisco, June 2010.

[3] TARTE, Y.-AMANNA, A.-OKWUDIAFOR, C.: Experimental Testbed for Investigating IEEE 802.11 Handoff in Vehicular Environment, Proc. of the IEEE SoutheastCon, Concord, March 2010.

[4] TERZIYAN, V.-KAYKOVA, O.-ZHOVTOBRYUKH, D. UbiRoad: Semantic Middleware for Context-Aware Smart Road Environments: Proc. of the $5^{\text {th }}$ International Conference on Internet and Web Applications and Services (ICIW), Barcelona, May 2010.

[5] CHEN, W.-GUHA, R. K.-CHENNIKARA-VARGHESE, J. -PANG, M.-VUYYURU, R. K.-FUKUYAMA, J. : Context-Driven Disruption Tolerant Networking For Vehicular Applications, Proc. of the IEEE Vehicular Networking Conference (VNC), Jersey City, Dec 2010.

[6] RAWAT, D. B.-TREeUMnUK, D.-POPESCU, D. C.ABUELELA, M.-OLARIU, S. : Challenges and Perspectives in the Implementation of the NOTICE Architecture for Vehicular Communications, Proc. of the $2^{\text {nd }}$ IEEE Workshop on Mobile Vehicular Networks (MoVeNet), Atlanta, Sep 2008.

[7] POPESCU, D. C.-TREEUMNUK, D.-OLARIU, S.: Requirements for the Physical Layer of the NOTICE System for Vehicular Communications, IEEE GLOBECOM Workshops, Honolulu, Nov-Dec 2009.

[8] YU, T. H.-MOON, Y. S.-CHEN, J.-FUNG, H. K.-KO, H. F.-WANG, R.: An Intelligent Night Vision System for Automobiles, Proc. of the $11^{\text {th }}$ IAPR Conference on Machine Vision Applications, Yokohama, May 2009..

[9] CHUI, M.-LÖFFLER, M.-ROBERTS, R. : The Internet of Things, McKinsey Quarterly No. 2 (2010), 1-9.

[10] BOYS, J. T.-GREEN, A. W. : Intelligent Road-Studs - Lighting the Paths of the Future, IPENZ Transactions 24 No. 1/GEN (1997), 33-41.

[11] GRAHAM-ROWE, D.-DANGER, H. A.: The Studs that Mark Out Road Lanes at Night are Getting Smarter, New Scientist 160 No. 2160 (Nov 1998).

12] KNAIAN, A. N.: A Wireless Sensor Network for Smart Roadbeds and Intelligent Transportation Systemsookinfo MEng thesis, Massachusetts Institute of Technology, June 2000.

[13] Highways agency, www.highways.gov.uk, accessed June 27, 2011.

[14] Astucia Traffic safety systems, www.astucia.co.uk, accessed June 27, 2011.

[15] SAMARDŽIJA, D.-KOVAČ, E.-ISAILOVIĆ, D.-MILADINOVIĆ, B.-TESLIĆ, N.-KATONA, M. : Road Nail: Intelligent Road Marking System Testbed, Proc. of the IEEE Vehicular Networking Conference (VNC), Jersey City, Dec 2010.

[16] HOLFELDER, W.: Vehicle-to-Vehicle and Vehicle-to-Infrastructure Communication: Recent Developments, Opportunities and Challenges, Workshop: Future Generation Software Architectures in the Automotive Domain, La Jolla, Jan 2004.

[17] MOTSINGER, C.-HUBING, T. : A Review of Vehicle-to-Vehicle and Vehicle-to-Infrastructure Initiatives, Technical Report CVEL-07-03, The Clemson University Vehicular Electronics Laboratory, 2007.

[18] SANTA, J.-GOMEZ-SKARMETA, A. F.-SANCHEZ-ARTIGAS, M. : Architecture and Evaluation of a Unified V2V and V2I Communication System Based on Cellular Networks, Computer Communications 31 No. 12 (July 2008), 2850-2861.

[19] Parallax 555-28027, Product Documentation, Parallax Inc., v1.4, Sep 2009. 
[20] Intersil ISL29003, Light-to-Digital Output Sensor with High Sensitivity, Datasheet, Intersil Corporation, Aug 2008.

[21] VAN TREES, H. L.: Detection, Estimation, and Modulation Theory, Part I, Wiley, New York, 1968.

[22] Texas Instruments CC2530, A True System-On-Chip Solution for 2.4-GHz IEEE 802.15.4 and ZigBee Applications, Datasheet, Texas Instruments Inc., Apr 2009.

[23] IEEE Std 802.15.4 - 2003, IEEE Computer Society, Oct 2003.

[24] SOL4 Velleman. Valleman Inc.

[25] IPv6 over Low Power WPAN (6LoWPAN), IETF Work Group, Dec 2008

[26] Link Layer Topology Discovery Protocol Specification, Microsoft Corporation, Sep 2006.

[27] BREITBART, Y.-GAROFALAKIS, M.-JAI, B.-MARTIN, C.-RASTOGI, R.-SILBERSCHATZ, A.: Topology Discovery in Heterogeneous IP Networks: the NetInventory System, IEEE/ACM Transactions on Networking, 12 No. 3 (June 2004), 401-414.

[28] HOWLAND, P.: Editorial - Passive Radar Systems, IEE Proceedings: Radar, Sonar and Navigation 152 (June 2005), 105-106.

Received 6 July 2011

Dragan Samardžija received the BSc degree in electrical and computer engineering in 1996 from the University of Novi Sad, Serbia, and the MSc and PhD degrees in electrical engineering from Wireless Information Network Laboratory (WINLAB), Rutgers University, USA, in 2000 and 2004, respectively. Since 2000 he has been with Bell Laboratories, Alcatel-Lucent, where he has been involved in research in the field of the next generation wireless systems. He is an assistant professor at the Computing and Control Department, University of Novi Sad. He authored more than 50 journal and conference publications. He has been a participant in a number of research projects sponsored either by the NSF or the European Union. As a member of the BLAST project team, he is a recipient of the 2002 Bell Labs President's Award. He received Central Bell Labs Teamwork Award for the HSDPA Demonstration Team, 2003.

Nikola Teslić received the BSc, MSc and PhD degrees in electrical engineering from the Faculty of Technical Sciences, University of Novi Sad, in 1995, 1997 and 1999, respectively. $\mathrm{He}$ is a professor at the Computing and Control Department,
University of Novi Sad. He is the CTO of the RT-RK Company, delivering development services and products in consumer area. His research interest covers design of real time systems, audio and video processing and design of testing systems.

Branislav M. Todorović was born in Belgrade, Serbia, in 1959. He received Dipl Eng and MSc degrees from the Faculty of Electrical Engineering, University of Belgrade, and $\mathrm{PhD}$ degree from the Faculty of Technical Sciences, University of Novi Sad, in 1983, 1988 and 1997, respectively. He is a senior research fellow at the RT-RK, Institute for Computer Based Systems, and an associate professor at the Military Academy, University of Defense, Belgrade. Prior to joining RT-RK, he was with the Institute of Microwave Techniques and Electronics IMTEL, Centre for Multidisciplinary Research, and the Military Technical Institute (VTI, Institute of Electrical Engineering) in Belgrade. His research interests are in the wide area of radio telecommunications and digital signal processing. He has authored or coauthored about 100 peer-reviewed journal and conference papers and two books.

Erne Kovač received the MSc degree in electrical engineering from the Faculty of Technical Sciences, University of Novi Sad, in 2010. He holds position of an R\&D associate at the RT-RK, Institute for Computer Based Systems. His research interests include design of embedded systems, wireless networks and renewable energy systems.

Đorde Isailović received the M.Sc. degree in electrical engineering from the Faculty of Technical Sciences, University of Novi Sad, Serbia, in 2010. Since then, he has been with the RT- RK, Institute for Computer Based Systems, where he is currently an R\&D associate. His main areas of research interests include design and analysis of various sensor systems in different wireless networks.

Bojan Miladinović received the BSc degree in electrical engineering from the Faculty of Technical Sciences, University of Novi Sad, in 2011. He holds position of a junior R\&D associate at the RT-RK, Institute for Computer Based Systems. His research interests include design of embedded systems and wireless networks.

*** This work was partly presented at the IEEE Vehicular Networking Conference (VNC), Jersey City, Dec. 2010.

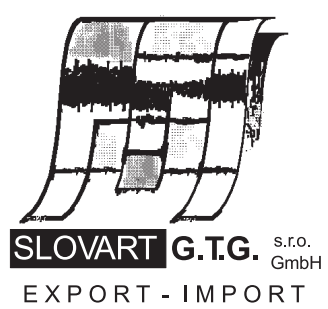

EXPORT - IMPORT
E X P OR T - I M P OR T

of periodicals and of non-periodically printed matters, books and CD-ROMs

Krupinská 4 PO BOX 152, 85299 Bratislava 5, Slovakia tel: ++421 263839 472-3, fax: ++421263839485 info@slovart-gtg.sk; http://www.slovart-gtg.sk

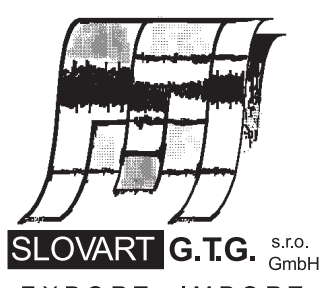

EXPORT - IMPORT 